\section{Fatal iron toxicity in adults}

Iron is an essential element for normal cellular function, but in excess it is highly cytotoxic, and can even be lethal, as it is a systemic intracellular poison. ${ }^{1}$ Multiple formulations of iron are available in the market to correct anemia in the form of injections, tablets and syrups. Iron poisoning is most commonly seen in children but rarely in adults. ${ }^{2,3}$ Among adults, it has been described as accidental overdoses (mainly in pregnancy) and as suicidal attempts. ${ }^{4}$ Clinical outcome is variable and depends on the quantity of iron ingested, other drugs ingested and the time to receive treatment. ${ }^{3}$ We report two cases: The first, of an 18-year-old girl who, despite our best efforts, died from intra-cranial hemorrhage 1 week following the ingestion of iron tablets and the second, the case of a 33 year old who recovered after receiving timely intervention following early presentation. The authors would like to draw attention to the diverse presentation of iron toxicity in patients and to stress on the fact that early intervention is an important prognostic factor.

\section{Case Report 1}

An18 year old female presented after alleged suicidal ingestion of 50 tablets of Iron amounting to $64 \mathrm{mg} / \mathrm{kg}$. She developed nausea, vomiting and abdominal discomfort, and was taken to a Primary Health Centre where gastric lavage and supportive treatment was given. Seventy two hours after ingestion, she developed jaundice and gradual worsening of sensorium. She reported to our hospital on day 4 of ingestion. At presentation her pulse rate was 90/min, BP 90/60mmHg, RR 22/min and $\mathrm{SpO} 294 \%$ on room air. She had mild icterus and pedal edema. Shewas drowsy with GCS E3V4M5, pupils B/L equal, reacting to light and flexor plantars.

Her lab Investigations at admission showed the following: RBS $28 \mathrm{mg} / \mathrm{dl}, \mathrm{Hb} 8.6 \mathrm{~g} / \mathrm{dl}$, TLC $14,500 / \mathrm{cu}$ $\mathrm{mm}$, Platelets 3.36lac/cu mm,s. creat $0.71 \mathrm{mg} \% \mathrm{PT} / \mathrm{INR}$ No Clot,s.bilirubin $4.38 \mathrm{mg} \%$, direct bilirubin $1.95 \mathrm{mg} \%$, AST 2235U/L, ALT 4611U/L, ALP 187U/L, pH 7.06, HCO3 6.2, Lactate $>15$ and arterial NH3 272. Her serum iron level was $110 \mu \mathrm{g} / \mathrm{dl}$, TIBC $409 \mu \mathrm{g} / \mathrm{dl}$ and ferritin 606.5ng/ ml. USG Abdomen and X-Ray chest were normal.
Emergency treatment was started in the form of iron chelation therapy with desferrioxamineat dose of $15 \mathrm{mg} / \mathrm{kg} / \mathrm{hr}$ along with $\mathrm{N}$-acetyl cysteine and inotropic support.In view of hepatic encephalopathy high bowel wash with lactulose, rifaximin and L-Ornithine L-Aspartate was provided.Four units of Fresh Frozen Plasma (FFP) were transfused and patient was shifted to ICU for further management. On 2nd day of admission, her sensorium worsened and she developed respiratory distress. She was intubated and placed on ventilator support.Her inotropic support had to be increased(both dopamine and noradrenaline). 2DEcho was done to rule out cardiogenic shock. Her repeat lab investigations showed PT/INR $6.2, \mathrm{Hb} 8.2 \mathrm{~g} / \mathrm{dl}$, APC $2.41 \mathrm{lac} / \mathrm{cu}$ mm,s.creat $0.92 \mathrm{mg} \%$,s. bilirubin $8.36 \mathrm{mg} \%$, Direct bilirubin2.4mg\%, AST 1536 U/L,ALT 4611U/L, ALP 187 U/L,NH3 242.

Single donor plasmapharesis was given along with 2 more units of FFP.Her sensorium continued to deteriorate despite all efforts and she developed asymmetric pupils (right pupil constricted, nonreacting;leftpupil was dilated and non-reacting) GCS was E1VtM1, plantars were up going.Non Contrast CT of the head showed a large thalamic bleed with midline shift and herniation. She succumbed, despite all efforts. Our final diagnosis was acute liver failure secondary to iron overload complicated with multiorgan failure and coagulopathy.

\section{Case Report 2}

A 33 year old lady allegedly consumed 60 tablets of ferrous sulphate amounting to total dose of $64 \mathrm{mg} / \mathrm{kg}$. She was immediately taken to a local hospital where gastric lavage was done and was rushed to our hospital. At presentation she was thermodynamically stable with normal pulse rate, $\mathrm{BP}$ and $\mathrm{O} 2$ saturation. Her lab investigations at admission showed $\mathrm{Hb} 12.3 \mathrm{~g} / \mathrm{dl}$, TLC 6400/cu mm, APC 3.61lac/cu mm, s.Bilirubin 1.3mg\%, ALT 33U/L, AST24U/L,PT/ INR 0.94, s. iron $68 \mu \mathrm{g} / \mathrm{l}$, TIBC $387 \mu \mathrm{g} / \mathrm{l}$, s. ferritin 585ng/ $\mathrm{ml}$. USG abdomen and X-Ray chest were normal.

Immediate iron chelation therapy with desferroximine was begun with $1 \mathrm{~g}$ stat, followed by an infusion started at a dose of $15 \mathrm{mg} / \mathrm{kg} / \mathrm{hr}$. She was transferred to the HDU for further management. She 
remained stable and showed no signs of deterioration with normal results on all follow up investigations. Chelation was stopped after 24 hours and only supportive treatment was continues. She was discharged in stable condition on day. $^{5}$

\section{Discussion}

Iron, by mass is the most abundant trace element in the body and is essential in most biological systems for its use in transport of oxygen and cellular respiration. Iron poisoning can either be accidental or suicidal. In case of an overdose, iron causes corrosive damage to the gastrointestinal (GI) mucosa and can lead to acute hemorrhagic gastritis, massive fluid loss (because of third spacing), bleeding and shock, which also results in massive iron absorption. ${ }^{5}$

Intracellularly, iron exerts toxic effects on the mitochondria leading to uncoupling of oxidative phosphorylation. It leads to anaerobic metabolism and subsequently metabolic acidosis. Iron also causes increased capillary permeability, coagulopathy and arteriolar dilatation leading to severe acidosis and shock. ${ }^{2}$ Oxygen free radicals cause myocardial damage and can induce cardiac failure. It can cause pulmonary damage, kidney injury, pancreatitis and peri-portal hepatic necrosis. ${ }^{6}$

Baranwal and Singhi described the clinical manifestations in children and divided them into 4 stages. Stage-I (Stage of gastrointestinal toxicity): GI effects predominate althoughin severe cases central nervous system depression and cardiovascular collapse can also occur. Stage-II (Stage of apparent stabilization or quiescent phase): The apparent stabilization is said to be due to redistribution of free circulating iron from intravascular space into reticuloendothelial cells and intracellular compartment. Stage-III (Stage of mitochondrial toxicity): Besides hepatic injury, acute tubular necrosis, pulmonary hemorrhage, and acute respiratory distress syndrome may occur at this stage. Stage-IV (Stage of gastric scarring): This may occur after 2-6 weeks of acute poisoning in very severe cases and usually present with recurrent vomiting secondary to gastric outlet obstruction. ${ }^{6}$

Iron poisoning causes hepatotoxicity as a primary insult and the level of damage correlates to the serum iron concentration. Robertson et al, in their study defined iron poisoning as a serum iron concentration $>300 \mu \mathrm{g} / \mathrm{dL}$ (55 $\mu \mathrm{mol} / \mathrm{L}$ ) within 12 hours of ingestion. Hepatotoxicity was defined as a serum transaminase (either ALT or AST) $>150$ U/L while severe hepatotoxicity was defined $>1000 \mathrm{U} /$ L7.This was seen in our first case. The serum iron levels can be unreliable in late presentation of the patient since the iron is re-distributed intra-cellularly.

Treatment modalities include decontamination with gastric lavage and iron chelation therapy.Gastric lavage is usually confined to those who present within one hour of ingestion and those who have ingested more than $20 \mathrm{mg} / \mathrm{kg} \cdot{ }^{1,2,7}$ Desferrioxamine is the only approved iron chelator available and administered as a continuous intravenous infusion at $15 \mathrm{mg} / \mathrm{kg} / \mathrm{hr} .2,7$ Administration of desferrioxamine after acute intoxication may color the urine a pinkish red, a phenomenon termed "vinrose urine". ${ }^{3}$ Disappearance of the 'vin rose' discoloration has often been taken as an endpoint to iron chelation. $\mathrm{N}$-acetyl cysteine is used when hepatotoxicity is evident in pure ferrous sulphate overdose. ${ }^{2}$ All of these agents were used in our two patients in the mentioned dosage and we were able to get good results in our second patient who had presented early.

RISHU BHANOT ${ }^{1}$ N.P. JAIN ${ }^{1}$ VANDANA MIDHA ${ }^{1}$ SHWETA GUPTA ${ }^{2}$

\author{
${ }^{1}$ Department of Internal Medicine \\ ${ }^{2}$ Department of Obstetrics and Gynecology, \\ Dayanand Medical College and Hospital, \\ Ludhiana, Punjab, India \\ Corresponding Author: Dr. RishuBhanot \\ Email:drbhanotrishu@gmail.com
}

\section{References}

1. Skoczynska A et al.Acute iron poisoning in adult female. Human and Experimental Toxicology. 2007;26:663-66.

2. Mahesh KM, Rani R. CASE OF IRON POISONING- 
CASE REPORT. International Journal of Basic and Applied Medical Sciences. 2014 Vol. 4 (3), pp. 101-103.

3. Abhilash KPP, Arul JJ, Bala D. Fatal overdose of iron tablets in adults. Indian J Crit Care Med. 2013;17(5): 311-3..

4. Jover F, Andreu L, Robert J, Merino J. [Acute iron poisoning. A case report]. Rev Med Chil. 2001;129(6): 660-2.

5. Baranwal AK, Singhi SC. Acute iron poisoning: Management guidelines. Indian Pediatr. 2003;40:534-40

6. Daram SR and Hayashi PH. Acute liver failure due to iron overdose in an adult. Southern Medical Journal. 2005; 98: 241-44.

7. Robertson A, Tenenbein M. Hepatotoxicity in acute iron poisoning. Hum ExpToxicol. 2005;24:559-62.

\section{Quetiapine induced acute pancreatitis: a case report from Eastern Nepal}

Acute pancreatitis is an inflammatory condition of the pancreas with variable involvement ofextrapancreatic organs. Gallstones, alcohol and hypertriglyceridemia are the most commonly recognized etiologies of acute pancreatitis. Medications, autoimmune pancreatitis and ERCP (Endoscopic Retrograde Cholangiopancreatography) pancreatitis, apart from other rare causes, have also been implicated in the causation of the disease. Second-generation antipsychotics (SGAs) such as Quetiapine, olanzapine and risperidone, have well known metabolic side effects such as hyperlipidemia and hyperglycemia. ${ }^{1}$ Quetiapine is a widely used antipsychotic in the treatment of delirium. Although second-generation antipsychotics are associated with pancreatitis, Quetiapine-associated pancreatitis is extremely rare. ${ }^{2}$
We present what we believe to be the first report of Quetiapine induced acute pancreatitis seen at our tertiary care hospital in Eastern Nepal.

\section{Case Report}

A 32 year old male presented to the emergency room with progressive, moderate to severe epigastric pain for 3 days associated with nausea and post prandial bloating. The pain radiated to the back and right shoulder. He reported no history of fever, jaundice, vomiting of blood or dark stool. The patient reported no history of smoking or alcohol use and he consumed a vegan diet. He recently survived a major road traffic accident one and a half months ago, where he sustained a head concussion for which he was hospitalized and kept in the ICU for observation. During the hospital stay the patient developed delirium and was diagnosed to have depression on detailed psychiatric assessment. He was put on Quetiapine $25 \mathrm{mg}$ twice daily along with midazolam $5 \mathrm{mg}$ at bed time. He was on the atypical antipsychotic for one and half months before presenting with this episode of abdominal pain.

On examination he was in distress with abdominal pain. He had generalized tenderness, guarding and rigidity of the abdomen. He had not passed flatus for 3 days and his bowel sounds was sluggish. He was kept NPO (Nil per Oral),with a nasogastric tube, started on intravenous opioids and was given judicious hydration while closely monitoring his urine output. His investigations revealed high amylase $920 \mathrm{IU} / \mathrm{ml}$, lipase $1010 \mathrm{U} / 1$, hematocrit $42.5 \%$, total leukocyte count 17000 cells $/ \mathrm{mm} 3$ and hsCRP (high sensitivity C-reactiveprotein) $198.4 \mathrm{mg} / 1$. There was no evidence of hypertriglyceridemia, diabetic ketoacidosis or other metabolic abnormalities. His abdominal CT revealed moderate severity of the disease (Modified CTSI score- 4) with minimal left sided pleural effusion.

With the temporal relationship of use of Quetiapine for 6 weeks and the absence of typical etiological factors (eg. Alcohol, gallstones, hypertriglyceridemia) Quetiapine was implicated for the patient's acute pancreatitis. Psychiatric consultation was done and Quetiapine was stopped and replaced with escitalopram. The patient became medically stable after 3 days of hospitalisation. 